$\xi=-1$

\title{
Dynamic Cluster Based Routing Protocol with Sink Mobility Support for Underwater Wireless Sensor Networks
}

\author{
Anandalatchoumy $\mathbf{S}^{1 *}$, Sivaradje G \\ ${ }^{1}$ Research scholar, Pondicherry Engineering College. India. \\ ${ }^{2}$ Professor, Pondicherry Engineering College. India. \\ *Corresponding author E-mail: anandalatchoumys@pec.edu:
}

\begin{abstract}
Underwater wireless sensor networks are energy resource constrained due to the scarce battery capacity. Energy efficient routing protocol is highly demanded to be developed for such networks. It is indeed a challenging task to design routing protocol that can achieve energy efficiency due to the dynamic and harsh underwater environment. A dynamic cluster based routing protocol coupled with sink mobility support (DCMMS) is proposed. Two schemes are combined together in the protocol. One is the formation of clusters and two is the mobile sink management. The cluster formation includes cluster head election process and member association process. Each cluster member sends the sensed data to the cluster head. Multiple mobile sinks are deployed to gather data directly from cluster heads. Finally, mobile sinks send the collected data after proper aggregation to the static sinks located at the surface. Thus, sink mobility and the dynamic clustering technique together help to balance the load among the nodes thereby minimizing energy consumption to a significant extent and extending the network life span. Analytical simulations are extensively carried out to attest how the proposed protocol (DCMMS) achieves better performance with minimum energy consumption, less end to end delay and higher packet delivery ratio than its counterpart existing protocol (AA-RP).
\end{abstract}

Keywords: cluster formation; dynamic; energy consumption; sink mobility; underwater wireless sensor networks.

\section{Introduction}

A group of sensor nodes deployed underwater which communicate with each other through wireless acoustic communication channel to perform collaborative tasks in target region form underwater wireless sensor network (UWSN) [1]. The ability of the underwater sensor nodes to work in a collaborative manner in the network makes it possible to utilize UWSNs for a broad scope of underwater application domains like ocean monitoring, surveillance, disaster warning, detection oil reservoirs and mining resources of remote locations [2]. In UWSN, as the battery energy source of sensor nodes is scarce due to the limited capacity of battery power, the intelligent way of utilization of energy consumption becomes the key challenge to be considered while designing a routing strategy so that energy efficiency can be attained for a prolonged network span. As there exists many challenges posed by dynamic underwater acoustic channel such as high delay of acoustic signal propagation due to low and variable underwater sound speed varying from $1450 \mathrm{~m} / \mathrm{s}$ to $1530 \mathrm{~m} / \mathrm{s}$, high bit error rate, limited bandwidth, multi-path fading, etc. [3], the necessary efforts are required to be taken care of to mitigate them. Therefore, an intelligent strategy is demanded to be applied to the design of routing scheme for ensuring high energy efficiency. Energy efficiency can be achieved by load balancing among the nodes to enhance the network performance with prolonged network lifespan. Introduction of sink mobilization into the network causes the dynamic change in network topology with the passage of time. Mostly, sink mobility helps in improving load balancing resulting in balanced energy dissipation of nodes. Underwater nodes forward the information sensed from the detected events in the target area to the mobile sinks directly whenever mobile sinks become their in-range neighbors as a result of which traffic load on forwarding nodes can be reduced significantly and also communication void problem can be meticulously handled without the need to use separate void avoidance approach.

\section{Related Work}

To design the appropriate algorithms for the proposed protocol to meet the desired objective, information in the existing literature survey is analyzed. In [4], Yan H. et.al. developed the first localization free routing protocol for UWSNs which uses only depth information as the routing decision factor. The receiver node with depth less than that of sender node becomes an eligible next hop node to forward packet toward the sink in upward progress. introduced a reliable pressure based opportunistic routing for UWSNs called. In the HydroCast protocol developed by Noh Y. et.al. [5], nodes with higher rank of packet advancement and shorter holding time become the next most suitable node to forward packets toward the sink. The clustering technique is exploited in the routing procedure which aids in the enhancement of duplicate packet suppression. A void node is avoided by detouring path along the previous nodes to reach sink so as to reroute packet through the reestablished route. Jafri M. R. et.al. [6] developed a variant of DBR routing protocol called AMCTD which introduces the concept of making some of the nodes to be changed as courier nodes. Upon finding a courier node in its neighbor list, the 
forwarder node sends the received data packet to the courier node. An acknowledgement is sent by the courier node to suppress duplication of packets by other neighbor nodes. Otherwise, the node broadcasts the data packet to the remaining neighbor nodes. Noh Y. et. al. [7] proposed VAPR, a pressure and beacon based routing protocol. Each node periodically generates beacon packet and broadcast them to its one hop neighbor nodes. Each beacon packet consists of meta information like void node status, hop count and depth. Next hop node is selected based on beacon packet information. Nodes in the neighbor node list with true status will not participate in packet forwarding thereby aid in tackling the communication void problem.

In [8], GEDAR proposed by R. Coutinho W. L. et. al, built-in depth adjustment capability enabled circuitry is used to displace the void node in vertical direction. When void node occurs, its depth is adjusted forward data toward the sink.Wang Zhuo et. al. proposed a protocol which uses autonomous underwater vehicles (AUVs) for data collection from underwater sensor nodes [9]. In this scheme, a cluster is formed with one cluster head node responsible for data collection from the member nodes so that energy consumption is minimized. As multi-hop data transmission to AUVs is not incorporated, packets have to wait for a long time with a node till it locates AUV in its communication range. Additionally, communication void creation occurs due to immutable selection of cluster head node which results in degrading of the network performance.

\section{Overview of the Proposed Protocol}

Dynamic Cluster based routing protocol with sink mobility support is proposed and it is termed as DCMMS. The operation of the protocol has three phases:

(i) Cluster formation phase

(ii) Mobile sink management phase

(iii) Data forwarding and routing phase.

\subsection{Network Architecture}

Multiple mobile sink architecture used for the implementation of the proposed protocol is shown in Fig. 1. In this architecture, sensor nodes and mobile sinks are deployed randomly underwater in three dimensional network fields along with set of static sinks located at the water surface. The sensor nodes can detect and sense the events and transmit them to single-hop neighbor nodes. Mobile sinks are the sensor nodes built with more capabilities than that of ordinary sensor modes. That is, they are provided with more memory, rechargeable battery and high power acoustic modem so as to gather data directly from the nodes and buffer them. Additionally, mobile sinks are equipped with depth adjusting capable device. The static sinks have both acoustic modem and radio modem. Inter node communication of static sinks with underwater nodes and mobile sinks is through the use of acoustic modem and radio modem is meant to disseminate gathered data to the offshore data processing center.

\subsection{Cluster Formation Phase}

In this phase, a node with residual energy greater than threshold and the data storage buffer size larger than that of the other nodes is elected as cluster head. After getting elected, cluster head broadcasts a beacon message advertising its cluster head elected notification. Upon hearing this, the non-cluster members initiate joining request to the elected cluster head so that they become members associated with it. Thus, clusters are formed. Then, the cluster head instructs the members to follow the time-slot schedule generated to send the data in the collision-free manner.

\subsection{Mobile Sink Management Phase}

The mobility model used by the mobile sink the random waypoint mobility model. The sink traverses from one position to another position to collect the data. At every position, sink stops for a predetermined time called pause time to gather data. The mobile sink broadcasts its presence to in-range neighbors. Upon hearing this, all cluster heads will start sending data to it either directly when they are in one-hop range or in a multi-hop fashion through intermediate cluster heads. After the pause time expires, mobile sink sends stop signal so that cluster heads will be able to stop data transmission till they hear beacons from another closest mobile sink. Then the mobile sink changes its speed and direction of travel randomly to update its coordinates to reach a new location. It repeats its operation.

\subsection{Data Forwarding and Routing Phase}

In this phase, sensor nodes are assumed to generate data packets at random. If a data packet is generated by a sensor node, then it is referred to as source nodes. If a packet is received by a sensor node from other nodes, then it is called forwarder node. Each cluster member node sends the data to cluster. Then, data can be forwarded to surface static sink in two ways. One way is direct forwarding where data packets are transmitted to any of the nearby surface static sinks directly either from mobile sinks or from cluster heads. The other is multi-hop forwarding where data packets are transmitted to any of the nearby surface static sinks in multihop fashion from mobile sinks through intermediate mobile sinks. When a void node occurs, a sends a notification message of its void status. Upon receiving this information, mobile sink travels to the void node location and aid the void node to deliver its sensed data.

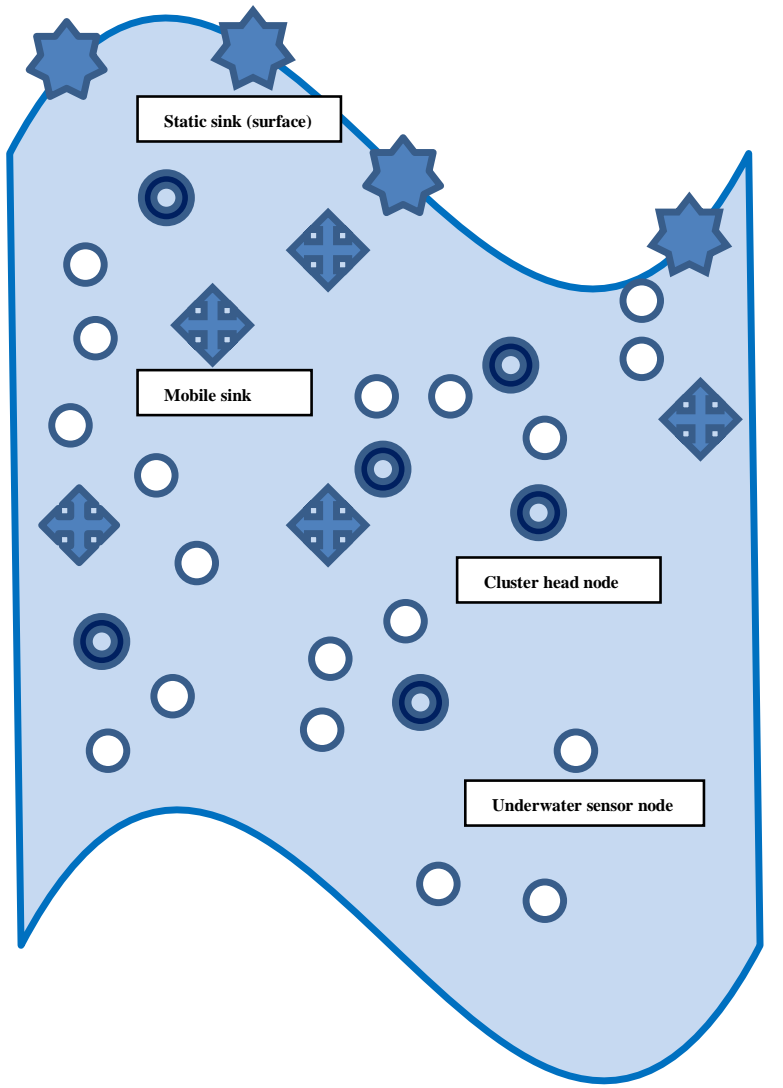

Fig. 1. Multiple mobile sink architecture 


\subsection{Algorithm for Data Forwarding and Routing}

Notations used:

MS -Mobile sink;

MS_RC -Transmission range of MS;

CH_ne - neighbor cluster heads of MS

$\mathrm{CH} \_\mathrm{Nr}$ - neighbor cluster head of ne;

CH_ne_rc - Transmission range of $\mathrm{CH} \_$ne

vn: void node

vn_nmsg-voidnode_notification_message

The steps for data forwarding from cluster heads to in-range mobile sink are detailed below:

Step 1: MS broadcasts hello packet periodically and waits for pause time

Step 2: $\quad \mathrm{CH}_{-}$ne receives the hello packet

Step 3: if dis $(\mathrm{CH}$ _ne, MS $)<\left(\mathrm{CH} \_\right.$ne_rc $)$

Step 4: $\quad$ CH_ne sends data to MS

Step 5: $\quad$ if (vn_nmsg is recd by MS) MS changes its position

MS broadcasts hello packet

vn sends data to MS

go to step 1

else

MS remains till pause time.

Repeat step 4 with next neighbor end if

else

Step 6: CH_ne broadcasts packet to $\mathrm{CH} \_\mathrm{Nr}$

Step 7: CH_ne -> CH_Nr

go to step 3

end if

\section{Simulation Results}

Simulations are carried out with appropriate values assigned to simulation parameters as detailed in Table. 1. Simulation results of the proposed work (DCMMS) for varying density of nodes are presented against the existing AA-RP. The performance is evaluated against three essential performance metrics:

(i) Energy consumption

(ii) End to end delay

(iii) Packet delivery ratio

\subsection{Performance Metrics Analysis}

Energy consumption:

The energy consumption comparison is shown in Fig. 2. It can be observed that proposed scheme (DCMMS) outperforms the compared scheme of AA-RP in the graph. The reason for significant reduction of energy consumption is that nodes forward data only to the cluster heads which in turn forward to the nearest mobile sink within the communication vicinity. Thus, number of transmissions is greatly reduced in dense networks resulting in higher energy efficiency.

\section{End to end delay}

In Fig. 3, it can be clearly seen that end-to-end delay is highly reduced in the proposed work. The reason is the positive effect of clustering approach along with sink mobility support in reducing number of transmissions when node density increases and the selection of optimal minimum path.

\section{Packet delivery ratio:}

The packet delivery ratio comparison is clearly shown in Fig. 4. The proposed work outperforms the existing scheme because of the fact that data collection by mobile sinks directly from nodes reduces packet loss to a great extent.
Table 1: Simulation parameters

\begin{tabular}{|c|c|c|}
\hline Parameter & \multirow{2}{*}{\multicolumn{2}{|c|}{$\begin{array}{l}\text { Value } \\
1500 \times 1500 \times 1500\end{array}$}} \\
\hline $\begin{array}{l}\text { Network } \\
\text { size }\end{array}$ & & \\
\hline \multirow[t]{2}{*}{ Initial energy } & node & Mobile sink \\
\hline & $100 \mathrm{~J}$ & $300 \mathrm{~J}$ \\
\hline \multirow{2}{*}{ Transmission range } & node & Mobile sink \\
\hline & $250 \mathrm{~m}$ & $1000 \mathrm{~m}$ \\
\hline Sensor nodes & \multicolumn{2}{|c|}{$100-500$ (in steps of 50) } \\
\hline Mobile sinks & \multicolumn{2}{|c|}{10} \\
\hline Static surface sinks & \multicolumn{2}{|l|}{45} \\
\hline Transmission power & \multicolumn{2}{|l|}{$2 \mathrm{~W}$} \\
\hline Reception power & \multicolumn{2}{|l|}{$0.1 \mathrm{~W}$} \\
\hline Idle power & \multicolumn{2}{|c|}{$10 \mathrm{~mW}$} \\
\hline Mobility model & \multicolumn{2}{|c|}{ Randomwaypoint } \\
\hline $\begin{array}{l}\text { Threshold } \\
\text { energy }\end{array}$ & \multicolumn{2}{|l|}{$10 \mathrm{~J}$} \\
\hline Data rate & \multicolumn{2}{|c|}{$10 \mathrm{kbps}$} \\
\hline
\end{tabular}

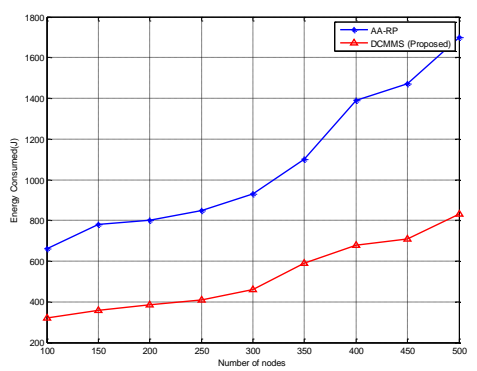

Fig. 2. Energy consumption comparison

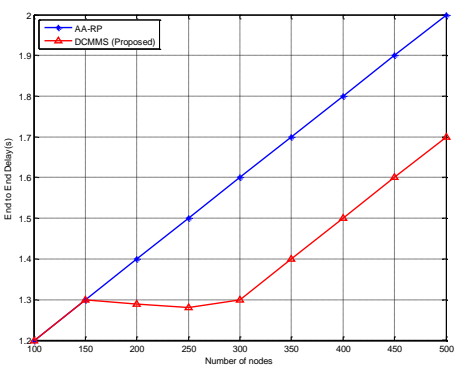

Fig. 3. End to end delay comparison

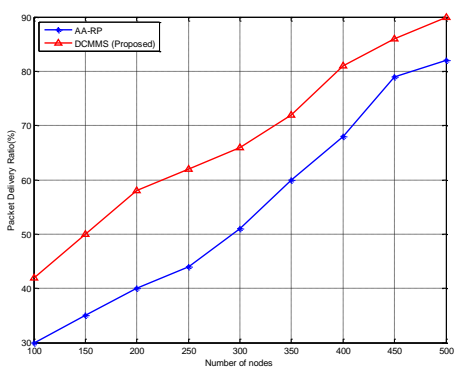

Fig. 4. Packet delivery ratio comparison

\section{Conclusion}

The proposed dynamic cluster based routing protocol with sink mobility support (DCMMS) has been designed for energy efficient data transmission to static sinks through mobile sinks. Multiple mobile sink architecture is used as network model. Dynamic clusters are formed grouping sensor nodes under a cluster head node. Cluster head of each cluster is re-elected upon attaining a threshold energy level rotationally to maintain load balancing among nodes. Cluster heads either directly send their buffered data to nearby mobile sink or forward the data to far away mobile sink through intermediate cluster heads at a shallower depth and at a shorter distance to mobile sink in a multi-hop fashion. End to end 
delay, energy consumption and packet delivery ratio are highly improved.

\section{References}

[1] Akyildiz, I.F., Pompili, D., Melodia, T. "Underwater acoustic sensor networks: $\quad$ Research challenges.",Ad Hoc Netw., 2005, 3 257-279.

[2] Vasilescu, I.; Kotay, K.; Rus, D.; Dunbabin, M.; Corke, P. "Data collection, storage, and retrieval with an underwater sensor network.",Proceedings of the 3rd International Conference on Embedded Networked Sensor Systems, San Diego, CA, USA, 2-4 November 2005; pp. 154-165.

[3] Climent S, Sanchez A., Capella J. V., Meratnia N., and Serrano J.J., "Underwater acoustic wireless sensor networks: Advances and future trends in physical, mac and routing layers," ,Sensors, vol. 14, no. 1, January 2014, pp. 795-833.

[4] Yan, H.; Shi, Z.J.; Cui, J.H. "DBR: Depth-based routing for underwater sensor networks.", Proceedings of the International Confer ence on Research in Networking, Singapore, 5-9 May 2008, pp. 72-86.

[5] Noh Y., Lee U., Wang P., Choi B. S. C., and Gerla M., "HydroCast:Pressure Routing for Underwater Sensor Networks,", IEEE Transactions on Vehicular Technology, vol. 10, no. 5, 2013, pp. $750-765$.

[6] Jafri M. R., Ahmed S., Javaid N., Ahmad Z. , and Qureshi R. "Amctd: Adaptive mobility of courier nodes in threshold-optimized dbr protocol for underwater wireless sensor networks," in Eighth International Con- ference on Broadband and Wireless Computing, Communication and Applications (BWCCA). Compiegne,France: IEEE, October 2013, pp. 93-99.

[7] Noh Y., Lee U., Wang P., Choi B. S. C., and Gerla M., "VAPR Void-aware pressure routing for underwater sensor networks," IEEE Transactions on Mobile Computing, vol. 12, no. 5, May 2013, pp. $895-908$

[8] Coutinho, R.W.L.; Boukerche, A.; Vieira, L.F.M.; Loureiro, A.A.F GEDAR: Geographic and opportunistic routing protocol with depth adjustment for mobile underwater sensor networks. In Proceedings of the 2014 IEEE International Conference on Communications (ICC), Sydney, Australia, 10-14 June, 2014, pp. 251-256.

[9] Wang Zhuo, Guo Hongmei, Jiang Longjie and Feng Xiaoning," AUV-aided communication method for underwater mobile sensor network:", OCEANS, 2016 , pp.1-7, doi: 1109/OCEANSAP.2016.74853 